\title{
Espaces « réels » et espaces virtuels. L'exemple des loisirs
}

"Real " and virtual space. The example of leisure

«Reale» und virtuelle Raüme. Das Beispiel der Freizeitgestaltung

\section{Hélène Haniotou}

\section{(2) OpenEdition \\ Journals}

Édition électronique

URL : http://journals.openedition.org/rge/4406

DOI : $10.4000 /$ rge. 4406

ISSN : $2108-6478$

Éditeur

Association des géographes de l'Est

Édition imprimée

Date de publication : 1 avril 1999

ISSN : 0035-3213

\section{Référence électronique}

Hélène Haniotou, «Espaces « réels » et espaces virtuels. L'exemple des loisirs », Revue Géographique de l'Est [En ligne], vol. 39 / 2-3 | 1999, mis en ligne le 04 septembre 2013, consulté le 08 septembre 2020. URL : http://journals.openedition.org/rge/4406 ; DOI : https://doi.org/10.4000/rge.4406

Ce document a été généré automatiquement le 8 septembre 2020.

Tous droits réservés 


\title{
Espaces « réels » et espaces virtuels. L'exemple des loisirs
}

\author{
"Real» and virtual space. The example of leisure \\ «Reale " und virtuelle Raüme. Das Beispiel der Freizeitgestaltung
}

\author{
Hélène Haniotou
}

"Quand l'automatisme sera général et que les bénéfices de la mécanisation seront socialisés, les hommes reviendront à l'état édénique qui existait dans les régions de production naturelle, comme dans les mers du Sud: le rite des loisirs remplacera le rite du travail, et le travail lui-même deviendra une sorte de jeu. Tel est le but idéal d'un système de production complètement mécanique et automatique : élimination du travail - avènement universel des loisirs» (L. Mumford, 1934).

1 Le débat sur la durée et la réduction du temps de travail est devenu plus qu'actuel ces dernières années avec une profusion d'ouvrages sur l'évolution historique ou future du travail, sur son organisation et sa nature, mais aussi sur sa «place» dans les sociétés actuelles occidentales. Nous ne traiterons pas ici de cette évolution, mais des conséquences d'une évolution éventuelle du temps de travail - temps et travail étant des notions qui, surtout depuis la révolution industrielle, forment une paire quasi indissociable - le temps étant devenu la « mesure » commune et commode par laquelle le travail est comptabilisé, évalué, enfin rémunéré. Une partie du résultat-bénéfice de cet "investissement" du temps humain, l'argent, est ensuite utilisé pour mettre en valeur ce qui, dans un sens, pourrait être considéré comme étant complémentaire au temps de travail : le temps libre, ou, de manière plus précise, le temps de loisirs ${ }^{1}$.

2 Les loisirs, que nous entendons comme étant l'ensemble des activités hors travail auxquelles les individus s'adonnent pour leur plaisir, semblent évoluer en parallèle avec le travail, influencés comme celui-ci par l'évolution technologique, aussi bien dans leur nature et leur "organisation" que dans leur durée et leur répartition dans l'année ; et cela dans la vie de chacun. En effet, l'expansion des nouvelles technologies ainsi que celle des réseaux modifient les rapports entre homme, espace et temps et donnent naissance à des «théories » sur les conséquences de ces modifications. Deux d'entre elles, qui semblent diamétralement opposées, sont celle de l'avènement d'une 
homogénéisation des espaces au niveau planétaire et à son opposé, celle de l'émergence des espaces marqués par de fortes disparités dues à l'inégalité de leur équipement.

La première, plus optimiste, qui correspondrait à l'expansion de toutes sortes de réseaux au niveau mondial, semble être la plus proche des tendances décelables actuellement dans les sociétés occidentales. Elle permet de faire des suppositions sur l'avenir et amène à poser des questions sur les possibilités d'évolution et d'organisation des espaces, si nous adhérons à l'idée de la diminution du temps de travail et, par conséquent, à celle de l'augmentation du temps de loisirs pour tous, dans un avenir (probablement lointain) pour lequel il n'est pas aisé de situer, actuellement, la ligne d'horizon.

Nous allons donc considérer - en anticipant - une future quasi-homogénéisation des accessibilités, créée grâce aux réseaux, par les réseaux-mêmes, qui rendrait les situations ${ }^{2}$ semblables, indifférentes, sur tout l'espace occupé (ou susceptible de l'être) par l'homme. Ceci signifierait que, indépendamment du lieu (du site), les mêmes possibilités d'accès (physique ou non) se présenteraient à toute personne; que l'accessibilité, due à la performance des réseaux physiques, tels les réseaux de transport, serait grandement facilitée; que l'importance de l'espace métrique ou kilométrique, «remplacée » petit à petit par le temps, dont la bonne gestion semble primordiale pour tout utilisateur de réseau.

\section{Réseaux et loisirs}

5 L'essor que connaissent les réseaux à l'heure actuelle - grâce à l'augmentation des vitesses - ainsi que les tendances d'utilisation du concept de réseau, dans l'organisation et la gestion, laissent deviner des bouleversements à venir, aussi bien dans le domaine social que dans le domaine spatial, qui est celui qui nous intéresse plus particulièrement. Une réseautisation mondialisée rendrait facile le raccordement de tout site, de tout point, si éloignés qu'ils soient des structures urbaines, offrant, à tout usager ou "consommateur", les mêmes possibilités d'utilisation, avec pourtant la contrainte de «solvabilité ».

6 Les réseaux étant des structures spatiales et temporelles voient ces dernières caractéristiques devenir de plus en plus dominantes dans la plupart de ceux qui se développent actuellement et qui sont, pour leur grande majorité, des réseaux de communication ou d'information (Fig. 1, A, B, C). D'une part, ces réseaux sont caractérisés par la possibilité qu'ils offrent de communiquer ou de s'informer en temps réel, d'autre part - et cela concerne tous les réseaux - il y a une "accélération " générale du mouvement des flux. 
Figure 1 : Transports et communications : deux logiques différentes. D’après P. Claval, 1995.
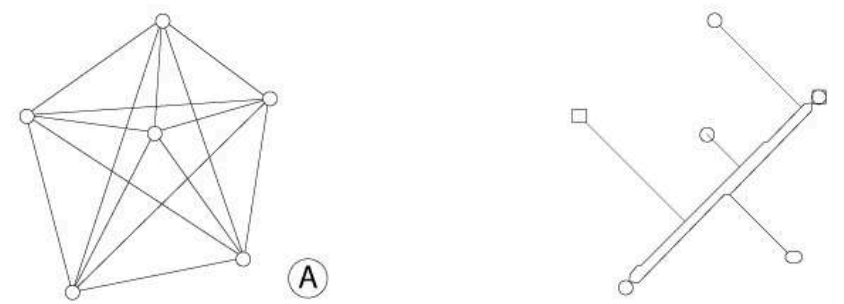

(B)

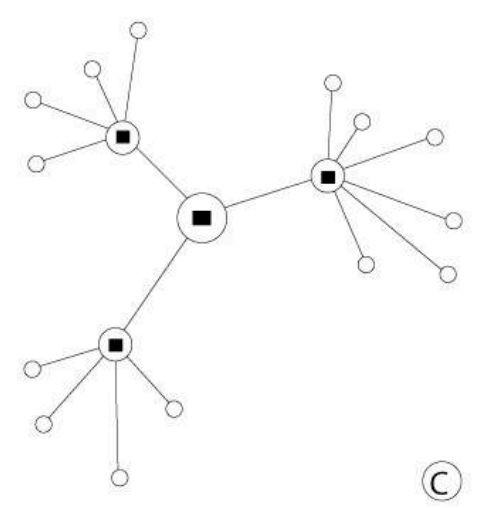

Logique de transport : (A) et (B) : une hiérarchie de lignes. Pour assurer le trafic des marchandises entre deux points. Le rattachement de tous les trafics (B) sur tout axe bien équipé est plus avantageux que le tracé de liaisons directes (A).

Logique de commercialisation : (C) : une hiérarchie de centres. Pour assurer l'écoulement des informations, la liaison de tous par le commutateur central est moins coûteuse que des liaisons directes qui multiplient la longueur des lignes et le nombre des standards. Lorsque les partenaires sont répartis sur une vaste étendue, la solution du commutateur unique allonge inutilement les lignes. En créant des commutateurs locaux reliés à un commutateur central, les trois totaux sont minimisés.

7 Aussi bien matériels qu'immatériels, les réseaux organisent et font se développer des « espaces » qui servent de support aux activités humaines, activités qui font partie de la sphère du travail ou du monde des loisirs. Ils créent également des espaces, spécifiques ou nouveaux, mais aussi des milieux ${ }^{3}$ (A. Berque, 1990), donc des relations nouvelles entre homme, espace, nature. Ils sont ainsi à la base d'un monde relationnel marqué aussi bien par l'interactivité (devenant parfois bien plus importante) que par l'interaction ${ }^{4}$. Ces nouveaux milieux - complémentaires pour A. Berque (1997) - peuvent être considérés comme ayant trait à deux types différents de relations: entre homme, "espace et nature (acception générale du terme) réels", ou bien entre homme, " espace et nature virtuels ». Ceci nous amène à considérer les loisirs sous deux angles différents, pas nécessairement opposés, les loisirs réels et les loisirs virtuels.

8 Nous allons parcourir d'abord assez brièvement ce que nous avons appelé « les loisirs réels » et les espaces correspondants, pour aborder ensuite le nouveau type de loisirs en pleine émergence. Générés par les réseaux d'information et de communication, ils donnent naissance à des pratiques inconnues précédemment dans une sphère d'action et d'activité cybernétique. 


\section{Loisirs réels} activités de loisirs, plus particulièrement du tourisme, à la fois par la diffusion des pratiques dans la société et par leur expansion géographique. Des efforts considérables ont été faits pour mieux organiser les espaces de loisirs et pour mieux vivre ses loisirs. Les loisirs, devenant non seulement une activité courante, banale, mais aussi une activité qui a tendance à s'étaler dans le temps, font sans cesse appel à l'innovation autant en ce qui concerne les lieux que les temps et les pratiques. L'évolution dans les pratiques des loisirs s'est accompagnée de l'évolution des espaces dans lesquels les gens vivent leurs loisirs, consomment leur temps de loisirs. Ainsi, des espaces de mouvement quotidien (rues, places) ou des espaces spécifiques (en partie) comme les villes thermales, on est passé à des espaces caractérisés par des structures propres, conçus comme des espaces de loisirs.

13 Depuis l'institution des congés payés ${ }^{5}$ surtout, les loisirs représentent une partie assez importante du temps humain quotidien, hebdomadaire ou annuel, ce qui a donné et continue à donner naissance à des services nouveaux, des organismes de loisirs - dont l'exemple le plus connu en France est le Club Méditerranée - mais également à des espaces de loisirs. Le développement des loisirs (des vacances, au départ) a eu pour conséquence l'aménagement et le développement des espaces - lieux d'accueil et d'attraction de ceux que nous avons appelés les "hommes en loisirs " (H. Haniotou, 1996). Ces espaces (réels) dans lesquels semblent se concentrer les flux de vacanciers ou cette partie des hommes en loisirs qui ont choisi de se déplacer, se trouvent le plus souvent au bord de la mer et à la montagne, de préférence dans des zones de climat 
tempéré et méditerranéen. La plupart des activités de loisirs ont toujours été liées au plein air et à la découverte de la nature, car elles représentaient une « fuite » (évasion) hors de la densité urbaine opprimante.

Les lieux de « consommation » de loisirs se détachent donc en général de la ville ou du lieu de résidence. Depuis les années 50, avec l'apparition des vacances organisées, des lieux qui leur sont spécifiquement destinés prolifèrent, tels les «villages » du Club Méditerranée, greffés sur les réseaux existants, dépendant des réseaux d'approvisionnement (en hommes, matière, énergie, information), mais en même temps constituant des espaces à part, dans le sens où tout ce qui se passe à l'intérieur est, ou peut être, sans relation "visible" avec l'extérieur. Leur fonctionnement peut être comparé à celui des «bulles» spatio-temporelles (J. de Rosnay, 1995) qui se différencient volontairement de leur environnement et du quotidien par leur « manière d'être ». L'attrait principal de ces "structures - lieux - espaces» de loisirs - qui pourrait être considéré comme celui qui marquera l'évolution des loisirs dans l'avenir - est la nature, mais aussi la culture et une certaine convivialité festive. Ces éléments répondent au besoin de «changement d'environnement ", à distance des villes, du lieu de résidence, même s'il ne s'agit que de distances courtes, spatialement ou temporellement parlant.

15 Par ailleurs, la possibilité de consommer son temps de loisirs sur place, sans qu'il y ait déplacement physique du consommateur, commence à apparaître, à se développer et donne lieu à des propositions d'évolution sociétale, selon lesquelles l'individu se déplacerait de moins en moins dans l'avenir, puisque toutes sortes de biens ou de services arriveraient directement à son domicile. Malgré l'attraction évidente des espaces réels et naturels, on peut déceler actuellement les premiers "germes " d'un loisir sur place, étant donné que le lieu où l'on «consomme » ses loisirs commence à devenir, pour certains, de moins en moins important. On pourrait même dire qu'après les vacances utopiques de l'après-guerre, se forment peu à peu des loisirs atopiques. Il s'agit de loisirs qui ne dépendent pas d'une certaine localisation ou d'un certain lieu, c'est-à-dire du site dans lequel ou à partir duquel on les vit; de loisirs qui, dans un certain sens, sont "ubiquitaires" (pantopiques), car on peut les avoir, les vivre partout et qui sont par conséquent - par l'intermédiaire des réseaux informatiques - non dépendants d'un lieu, au-dessus de tout lieu, donc hypertopiques.

Il est donc possible, en simplifiant, de ne parler que de deux types d'espaces de loisirs : des espaces réels, géographiques, dans lesquels sont "consommés » des loisirs " réels», et des espaces virtuels, à ancrage géographique mais "atopiques" - non pas par rapport à la localisation du consommateur, mais par rapport à celle de ces espaces qui, tout en étant atopiques par la quasi-impossibilité de les situer à l'intérieur des réseaux informatiques) sont en même temps "pantopiques » car au même instant, ils peuvent être localisés sur plusieurs « lieux » de ces réseaux - dans lesquels sont " consommés » des loisirs virtuels. Si, dans le futur, le temps de loisirs devait augmenter et si l'accessibilité des lieux ainsi que la solvabilité de chaque individu le permettaient, deux choix principaux se présenteraient pour chacun, par rapport au lieu et à la façon de consommer son temps de loisirs. On pourrait s'adonner à des activités de loisirs "virtuels », sans donc obligation de déplacement, ou bien choisir des loisirs " réels » dans des espaces réels avec changement de lieu, donc avec déplacement physique. 


\section{Loisirs virtuels}

Les loisirs virtuels apparaissent à la suite des loisirs imaginaires. Ces derniers ont été rendus possibles grâce à l'utilisation des appareils électroniques qui, depuis plus de vingt ans, envahissent notre quotidien, facilitent en général les tâches journalières, mais offrent également un loisir imaginaire. Les chaînes hi-fi, magnétoscopes et jeux vidéo, qui en font partie, sont déjà "en voie de dépassement ", remplacés par des produits nouveaux très performants, parfois considérés comme des «drogues électroniques » (J. de Rosnay, 1995), dont la place dans le quotidien augmente de plus en plus. Les nouvelles technologies et plus précisément les réseaux informatiques se trouvent à l'origine d'une nouvelle "nature », d'un nouvel « espace de liberté », faisant aussi émerger des loisirs d'un genre "inédit ", qui bouleversent les modes de vie et la manière de concevoir le temps et l'espace. Des loisirs imaginaires et électroniques provoquant une interaction entre le réel et l'imaginaire, créant un monde imaginaire dont nous restons "spectateurs", nous sommes passés aux loisirs virtuels qui, eux, créent un monde auquel nous pouvons participer comme acteurs, entrer et agir comme si nous en faisions partie.

18 À la base de ce monde virtuel, la réalité virtuelle ${ }^{6}$ - technique de communication, qui permet de "vivre " dans un environnement virtuel ou de se mouvoir dans un espace virtuel, connue aussi sous l'abréviation RV - doit son existence et son expansion à celle du réseau Internet ${ }^{7}$, souvent appelé le " réseau des réseaux " permettant toutes sortes de configurations relationnelles. Son utilisation amplifiée fait que l'on commence à lui attribuer le terme The Net (Le Réseau), dont les points terminaux sont formés par les couples usager-ordinateur. C'est le réseau par excellence de la réalité virtuelle, qui crée des "espaces » dans lesquels la personne connectée peut se déplacer et agir à l'aide d'appareillages spéciaux (casques, lunettes, gants, ...) permettant de manier ou de saisir des « objets » virtuels. Ainsi, plusieurs personnes, à partir de lieux différents, peuvent se "rencontrer" dans ce cyberespace ${ }^{8}$, se toucher, ou interagir. Ce sont des " voyageurs ", fixes et immobiles en réalité, qui créent leur propre espace de voyage et d'une certaine manière leur propre cosmos qui, par certains aspects, reste «immatériel». La virtualisation est considérée par P. Lévy (1995) comme un changement rapide, déstabilisant: "le mouvement même de "devenir autre" - ou hétérogenèse - de l'humain ». Bien qu'on l'oppose souvent au réel, le virtuel, pour ce même auteur, est plutôt l'opposé de l'actuel, car il «tend à s'actualiser » - c'est une manière d'être qui peut devenir actuelle - et il est caractérisé par son détachement d'ici et maintenant. On ne doit pas le confondre avec l'imaginaire puisque dans l'espace virtuel on "vit » par dédoublement: le corps tangible est présent dans un espace réel; le corps dédoublé, virtuel, peut être ici et là, ou partout à la fois, étant donné que dans le cyberespace n'importe quel point est, potentiellement, directement accessible de n'importe quel autre.

Le cyberespace constitue actuellement le marché le plus prometteur des décennies à venir et plusieurs entreprises de télécommunications «cherchent à se tailler des empires » (N. Negroponte, 1995). C'est un espace qui peut être "portable » grâce à un " costume de données » qui permet la création d'un environnement virtuel " portatif » dans lequel on peut « vivre » presque en permanence, si on le souhaite (P. Virilio, 1993). Il ne concerne pas uniquement le domaine des loisirs, mais aussi les domaines professionnel, commercial (cybershops) et éducatif ${ }^{10}$. Ainsi, l'informatique n'est plus 
une histoire d'ordinateurs, mais tend à devenir - si elle ne l'est pas déjà - un mode de vie (N. Negroponte, 1995).

Selon N. Negroponte « la véritable valeur d'un réseau [informatique] réside moins dans l'information qu'il transporte que dans la communauté qu'il forme». Des «tribus informatiques » se créent grâce à ces réseaux. Des groupes qui, selon Ph. Breton (1990), sont formés de personnes liées par un même système de valeurs, un même "code " social qui les isole des non-initiés à la «culture informatique ». Ces tribus sont à la fois peuplées d'hommes et d'ordinateurs; leurs membres ne considèrent pas les ordinateurs comme des simples machines, mais comme des interlocuteurs à part entière, sans pour autant voir en elles une sorte d'être humain. D'après ce même auteur, si les machines « séparent » les hommes, en même temps elles les rapprochent, mais elles les rapprochent autrement. Aux "machines de transport", par lesquelles on accédait à des espaces "réels", semblent donc succéder les " machines de communication " qui donnent accès aux espaces virtuels. La réalité virtuelle ainsi vécue peut être supplémentaire, de substitution ou d'appréhension de la réalité physique (même si elle ne peut pas toujours la remplacer ou se substituer à elle). Ce «règne prochain de la virtualité informatique", comme l'appelle P. Virilio (1993), n'aurait pas été possible sans la facilité de se connecter et de pouvoir communiquer avec tout point de la planète ayant accès aux mêmes réseaux (indifférence de situations).

21 L'espace virtuel est un espace créé par des réseaux, réels et virtuels en même temps. Leur réalité correspond à leur ancrage géographique, à des lieux précis que sont les couples usager-ordinateur, nœuds potentiels d'un réseau virtuel, créé lui, par les différentes configurations possibles et potentielles qui impliqueraient d'autres couples usager-ordinateur. Les réseaux virtuels (réseaux-service) correspondraient à la figure $\mathrm{A}$ où chaque nœud est (ou peut être) directement relié à chaque autre, en ayant cependant une "organisation" géographique (au sol) qui correspond à celle de la figure $\mathrm{C}$. On pourrait dire que ces réseaux n'existent qu'à travers une « noosphère » ou « infosphère " (souvent appelée par des auteurs de science-fiction datasphere, technocore ou metasphere) qui complète la géosphère (planète) et l'enrichit, tout en y ayant ses racines.

Rien ne peut - encore - confirmer que la réalité virtuelle ne sera recherchée qu'à partir du domicile. Elle pourrait très bien être combinée avec une "réalité réelle», ailleurs, à des endroits et lieux parfaitement équipés pour offrir le "maximum de sensations" à leurs "adeptes». Des endroits spéciaux de rencontre, permettant l'accès à ceux qui ne disposent pas de l'équipement nécessaire, existent déjà dans les grandes villes. Ce sont des endroits fréquentés surtout par les "initiés » et qui tiennent, tout en se différenciant, le rôle du café du village, lieu de communication, d'échange d'information et de recréation par excellence. Il s'agit des nœuds formés non pas par un couple, mais par plusieurs couples usager-ordinateur, des concentrations par conséquent, d'usagers-ordinateurs. Mais, pour le moment, "les utilisateurs de [ces] nouveaux objets techniques se recrutent surtout dans les classes moyennes et chez les jeunes; tandis que les personnes âgées, les femmes, les gens peu instruits ou de revenu modeste y accèdent difficilement » (V. Scardigli 1996).

Les situations (géographiques) indifférentes ne suffiraient donc pas pour que chacun ait accès à ces nouveaux services. L'indifférenciation de la solvabilité individuelle serait tout aussi indispensable. 


\section{Espaces et loisirs présents et à venir}

Les deux types de loisirs et d'espaces de loisirs que nous venons de définir s'opposent et se complètent, utilisant des réseaux physiques ou non. Si nous admettons que le temps futur prédominant sera celui de loisirs, que serait-il possible d'envisager par rapport aux espaces de consommation de ce temps? Il n'est possible de répondre à cette question que par des suppositions, fondées cependant sur des constatations ayant trait au passé et au présent, ainsi que sur des tendances qui, pour le moment, semblent se maintenir.

Si nous prenons l'exemple d'un organisme de loisirs actuel - le Club Méditerranée celui-ci est caractérisé par trois aspects essentiels: un aspect technologique («un ensemble d'infrastructures assurant la communication et la distribution des flux »), un aspect social (« un système relationnel entre des individus liés par les mêmes origines [...] ou par les mêmes intérêts ») et un aspect économique (" un modèle de partage des coûts entre plusieurs utilisateurs. Dans ce modèle, plus il y a d'utilisateurs, plus le coût marginal d'utilisation est bas, et donc plus la valeur économique du réseau est grande ») (Ch. Goldfinger 1994).

Le Club Méditerranée, connu surtout pour ses villages de vacances, a créé un univers de loisirs à la fois spécifique (au lieu géographique) et générique (par les éléments communs que l'on retrouve dans tous les « villages » ou espaces spécifiquement conçus et créés pour les loisirs) (Ch. Goldfinger, 1994). Ces espaces sont dotés de structures spatiales matérielles, «posées » dans des lieux ayant pour caractéristique principale la beauté naturelle exceptionnelle, attrait indubitable - du moins jusqu'à aujourd'hui des flux humains urbains. Une autre caractéristique, aussi importante que la première, est l'équipement qui désigne entre autres la "personnalité » de chaque espace, qui, elle, est fonction de la géographie et du climat local. Il est clair que l'équipement est aussi choisi en fonction des désirs et des besoins des hommes en loisirs. Cependant, ce qui différencie essentiellement ces espaces - mis à part leur « réalité »- des espaces virtuels est non seulement l'interaction humaine, mais également l'interdépendance entre clientèle et personnel et surtout la compétence des animateurs, leur savoir-faire et leur initiative, puisqu'ils doivent être en mesure de combler les éventuelles lacunes et de faire face à des imprévus de toute sorte ${ }^{11}$. Il existe donc un système relationnel d'interaction et non pas d'interactivité, en temps et espace réel.

Un organisme de loisirs passe un « contrat » avec ses clients, avec lesquels les diverses formes de " contact » ne sont que des supports complémentaires. Reste ensuite à créer la « connivence » nécessaire (celle de la confiance que l'on s'accorde mutuellement), pour son «maintien » et son fonctionnement. Avant de pouvoir vendre ses produits, un tel organisme doit se faire connaître auprès des futurs clients en passant par la publicité, par les brochures, par les agences etc., en utilisant donc des réseaux de communication pour faire circuler l'information. À partir du moment où le client contacte une agence, d'autres réseaux interviennent, comme par exemple celui de la gestion de réservations - qui doit non seulement informer la destination d'une arrivée éventuelle - mais également les compagnies de transport. Tout semble dépendre d'une multitude de réseaux en interrelation, qui font de chaque organisme de loisirs une vraie "machine relationnelle $»^{12}$ (A. Bressand et C. Distler, 1995 ). Il y a non seulement création de relations entre réseaux, mais ils existent grâce à ces relations, par ces relations-mêmes. 

potentielles se fait par un fonctionnement propre à « commutateurs ». Cette structure bien particulière est due au fait qu'il n'existe pas de circulation libre, mais des déplacements strictement orientés par les lieux de destination. La particularité de ce réseau répond à une logique et à un fonctionnement bien précis et propres à ce genre de déplacement (déplacement de loisirs à destination fixe). En effet, il ne s'agit pas de produits acheminés vers les clients qui les ont commandés, mais bien au contraire de déplacement des clients-mêmes vers les produits (destination) de leur choix (ce qui est l'opposé de la RV).

Si nous prenons encore l'exemple du Club Méditerranée, ses villages ont toujours été implantés ou développés dans des espaces interstitiels, c'est-à-dire dans des espaces qui n'étaient pas encore mis en valeur ou qui ne faisaient pas partie d'un « espace de mouvement " avant l'arrivée du Club. Ceci est valable pour tous les villages qui ont été créés par le Club, donc pour les villages les plus anciens (cette "stratégie» a été modifiée ces dernières années et consiste à récupérer des structures existantes et à les exploiter sans en être nécessairement propriétaire). Il est clair que de tels organismes doivent leur naissance, en grande partie, à celle des vacances pour les salariés et à l'augmentation du temps libre, celui « dégagé » du temps professionnel. Que les loisirs soient en nette augmentation depuis le début du siècle, sinon pour tous, du moins pour beaucoup de personnes, est également clair. Cependant, nous ne pouvons actuellement qu'affirmer la possibilité d'une augmentation future de ce temps de loisirs et par conséquent supposer le développement des réseaux de loisirs. organismes, pour une meilleure gestion des lieux et des flux, sont obligés de se constituer en réseau et de fonctionner en réseau. La canalisation, l'orientation et l'occupation des flux des hommes en loisirs est ce qui constitue leur qualité-atout, mise à part la qualité des services offerts sur place.

31 Certains réseaux du service « loisirs » doivent leur développement à ceux d'information et de télécommunications, à l'informatisation généralisée, mais aussi à l'essor des réseaux de transport. Ils existent grâce à l'interconnexion de diverses agences de loisirs, soit entre elles, soit entre ces dernières et des compagnies de transports ou des organismes de loisirs, hôtels, etc. La grande majorité de ces services sont localisées dans les grandes villes. S'il est facile de parler de réseau-service de loisirs, il devient bien plus difficile de parler de réseau-infrastructure de loisirs. Car si les infrastructures existent, en tant que structures spatiales matérielles, elles n'ont de relations qu'avec un certain « centre » et non entre elles. Il y a une sorte de dépendance « verticale » qu'il ne serait peut-être pas correct de caractériser de "hiérarchique ", étant donné que la dépendance du centre n'est pas totale. On pourrait dire que chaque espace de loisirs (village) fonctionne en unité indépendante, mais qui doit sa capacité de fonctionnement à une structure de contrôle qui n'intervient que pour le mettre en marche ou en cas de grave problème qui risquerait d'affecter le système entier.

Des réseaux de loisirs se constituent peu à peu, par tronçons. Certains leur appartiennent, d'autres sont empruntés aux réseaux existants. Ils se créent et occupent un espace propre que nous pourrions appeler espace interstitiel: interstitiel spatialement, interstitiel dans le temps ou les deux à la fois. Cet espace interstitiel espace inactif, ne faisant pas partie de l'espace réseautisé et informatisé, du moins au

Revue Géographique de l'Est, vol. 39 / 2-3 | 1999 
départ - se prête à accueillir des installations de loisirs, mieux que les espaces urbains, espaces matériellement moins flexibles, sinon saturés.

L'augmentation du temps de loisirs dans l'avenir pourrait ne pas conduire à plus de déplacements, à la conquête de l'espace, mais à la conquête du temps par la possibilité de se créer une "réalité virtuelle", chez-soi, sans qu'aucun déplacement ne soit nécessaire, comme nous l'avons déjà vu. Si nous considérons que le système de loisirs existe pour "produire du bonheur ", il appartiendra aux futurs hommes en loisirs de décider s'ils préfèrent les loisirs virtuels et atopiques aux loisirs réels et utopiques (en tant que «bulles » spatio-temporelles déconnectées de leur environnement) ou même topiques, que peuvent offrir les villages du Club Med.

En ce qui concerne les loisirs, les hommes en loisirs et leurs pratiques, on constate cependant que le tourisme, et les loisirs ensuite, ont évolué à la manière d'un système à "rétroaction positive " (où le plus entraîne le plus); nous considérons ici que cette évolution se poursuivra. Des loisirs-vacances caractérisés par un cycle annuel, nous passons à d'autres formes de loisirs fragmentés et étalés sur toute l'année. Les loisirs peuvent être à la fois quotidiens, hebdomadaires, mensuels ou annuels ; les activités de loisirs se modifient suivant l'évolution du goût des hommes en loisirs (en grande majorité urbains) qui ne cherchent plus à «bronzer idiots » mais ont des exigences d'ordre culturel, entre autres; pour s'offrir des loisirs, on doit disposer aussi bien de temps que d'argent. D'où une certaine complémentarité - nécessaire tant que le système reste le même - entre travail et loisirs ; les contraintes pécuniaires semblent être le critère principal pour tout choix de destination, en ce qui concerne les loisirs avec déplacement. Ainsi, non seulement les loisirs courts sont favorisés et en forte croissance, mais les lieux de loisirs se rapprochent des villes. Les nouvelles technologies n'influent pas seulement sur le fonctionnement et les rapports entre organismes de loisirs (et autres), mais elles sont entrées dans le quotidien, utilisées "à titre privé ". Des activités reliées à ces technologies sont également offertes par les organismes de loisirs. Il se pourrait très bien que dans l'avenir on puisse vivre son temps de loisirs en combinant le réel et le virtuel (loisirs virtuels dans des lieux naturels exceptionnels); enfin, un organisme de loisirs "international » est sujet aux fluctuations économicopolitiques des pays de destination. Il ne peut donc pas fonctionner dans un «monde » où la paix et la sécurité ne sont pas garanties. Il est également dépendant des réseaux d'infrastructures existants, sur lesquels il se greffe temporellement ou en permanence ou dont il utilise des parties. Il existe par conséquent un lien qui semble devenir de plus en plus étroit entre les technologies et les loisirs.

Si la possibilité de télécommuniquer, celle de pouvoir "se connecter " à tous les réseaux, est offerte dans les métapoles dont parle F. Ascher (1995) - théorie qui semble correspondre à celle de l'homogénéisation des espaces - il semblerait logique (mais pas exclusif) que les Métapolitains cherchent ailleurs des distractions, consomment ailleurs leur temps de loisirs. Si nous admettons que les deux types de loisirs - réels et virtuels - existeront au même titre dans un avenir lointain, pour que le choix du lieu de consommation du temps de loisirs soit véritablement libre pour chacun, ceci présuppose que les conditions sociales soient les mêmes pour tous, que la contrainte budgétaire n'existe plus pour personne. Les transformations spatiales dont nous avons parlé ouvrent des perspectives que notre économie marchande libérale, hautement technicisée, ne permet pas, si elle ne met pas au point d'autres règles de références d'acquisition, de répartition et de circulation monétaire. Si l'on admet que les 
situations, aussi bien géographiques que de solvabilité individuelle, deviendront identiques pour tous, il ne resterait que le goût personnel et la personnalité des sites qui joueraient le rôle de catalyseur dans ce choix. Ceci nous amène à supposer que seuls les sites « authentiques » caractérisés par leur «personnalité » naturelle et culturelle seraient assez attractifs pour justifier un déplacement physique de loisirs. Cette authenticité semble être la caractéristique qui pourrait faire face au virtuel, et qui deviendrait, par conséquent, une des causes principales de la revalorisation des espaces réels, due à leur « substance » naturelle, culturelle, historique, géographique.

Dans l'hypothèse donc de l'équipement égalitaire de la planète, le déplacement de loisirs dépendrait fortement du site, parce que, comme l'écrit F. Ascher (1995), «la qualité et la richesse d'un territoire tiennent à ce dont il dispose en propre et qui n'est ni télécommunicable ni transportable». En d'autres mots, si les situations deviennent indifférentes, avec les mêmes possibilités partout, ce qui devrait différencier un lieu de loisirs d'un autre, ce ne pourrait être que son site, ses qualités naturelles et culturelles hormis ses possibilités "technologiques"; les loisirs n'étant plus un besoin de consommation ou de valorisation par rapport aux autres, mais un droit et un besoin de « retrouver le sens du corps, de la nature, pour le bonheur et l'épanouissement personnels » (D. Clary, 1993).

Cette proposition reste ouverte et ne fait que présenter quelques premières idées et perspectives fondées sur une hypothèse qu'il n'est pas encore possible d'affirmer ou d'infirmer. Cependant, il nous a semblé intéressant de la présenter, afin d'ouvrir un débat sur l'avenir et sur les conséquences de l'offre croissante et fortement mondialisée des NTIC (Nouvelles Technologies d'Information et de Communication) face à la demande de sociétés encore très diversement solvables.

\section{BIBLIOGRAPHIE}

Ascher F. (1995). - Métapolis ou l'avenir des villes, Éditions Odile Jacob, 347 p.

Bailly A., Beguin H. ( 1990). - Introduction à la géographie humaine, Masson, Paris, 188 p.

Berque A. (1990). - Médiance de milieux en paysages, GIP RECLUS, 163 p.

Berque A. (1997). - « Biosphère ou cybermonde ? », Les cahiers de médiologie, n 3, Anciennes nations, nouveaux réseaux, Premier semestre 1997, Gallimard, pp. 75-81.

Bressand A., Distler C. (1995). - La planète relationnelle, Flammarion, 290 p.

Breton Ph. (1990). - La tribu informatique, Éditions Métailié, Paris, 191 p.

Clary D. (1993). - Le tourisme dans l'espace français, Masson, Paris, 358 p.

Claval P. (1995). - « Comment s'organise l'espace régional ?». Sciences Humaines, Hors série n 8 , février-mars 1995, pp. 6-9.

Corbin A. (1995). - L'avènement des loisirs. 1850-1960, Aubier, Paris, 471 p.

DATAR (1994). - La France au-delà du siècle, Datar/Éditions de l'Aube, 234 p. 
De La Baume R., Bertolus J.-J. (1995). — Les nouveaux maîtres du monde, Belfond, 237 p.

De Rosnay J. (1995). - L'homme symbiotique, Seuil, 350 p.

Goldfinger Ch. (1994). - L'utile et le futile. L'économie de l'immatériel, Éditions Odile Jacob, 622 p.

Haniotou H. (1996). - Loisirs généralisés et recomposition urbaine interstitielle, Presses Universitaires du Septentrion, Thèse à la carte, Villeneuve d'Ascq, $277 \mathrm{p}$.

Lévy P. (1995). - Qu'est-ce que le virtuel ?, Éditions La Découverte, Paris, 157 p.

Marseille J. (1996). - « Qui a inventé les congés payés ? ", L'Histoire, n 197, mars 1996, pp. 80-82.

Mumford L. (1934, 1950). - Technique et civilisation, Seuil, 415 p.

Negroponte N. (1995). - L'homme numérique, Robert Laffont, 296 p.

Scardigli V. (1996). - « Innovation et changement social », Sciences Humaines, n 59, mars 1996, pp. 20-23.

Virilio P. (1993). - L’art du moteur, Éditions Galilée, Paris, 199 p.

\section{NOTES}

1. Nous préférerons le terme de temps de loisirs, étant donné que le temps libre - qui peut être également utilisé comme temps de travail puisque son «remplissage » ne dépend que du libre choix des individus - ne serait pas une notion représentative de ce que nous allons développer ensuite.

2. Ce terme "évoque les relations de la ville avec un milieu physique extérieur plus lointain » tandis que le terme «site " est défini comme «les caractères morphologiques du milieu local d'implantation d'une ville » (A. Bailly et H. Beguin, 1990).

3. Un milieu est « la relation d'une société à l'espace et à la nature ».

4. Interactivité entre homme et machine, interaction entre hommes.

5. D'autres pays ont précédé la France (1936) dans ce domaine. Les congés payés ont été institués en 1905 en Allemagne ; en 1910 en Autriche-Hongrie, au Danemark et en Norvège ; entre 1919 et 1925 en Finlande, en Italie, en Tchécoslovaquie et en Pologne ; entre 1926 et 1934 au Luxembourg, en Grèce, en Roumanie, au Chili, au Mexique, en Espagne, en Suède, au Pérou, au Brésil, au Portugal (J. Marseille, 1996).

6. Le Japon est actuellement la pays le plus avancé dans les techniques du virtuel (DATAR, 1994). L'industrie de la simulation, en pleine expansion aujourd'hui, vise essentiellement le domaine des loisirs, et se trouve à la base de la création de la « réalité virtuelle » qui tend à devenir « un média de loisirs grand public» (N. Negroponte, 1995). Elle date de 1968 avec la fabrication du premier visiocasque par Ivan Sutherland (ibid.). Mais ce n'est que récemment, avec l'usage généralisé des ordinateurs, qu'elle commence réellement à se répandre.

7. Né aussi en 1968, initialement développé pour l'armée américaine, par la Defence Advanced Research Project Agency.

8. «Le cyberespace est le résultat d'un travail coopératif entre le moteur de réalité informatique du laboratoire et le moteur de réalité du cerveau » (H. Rheingold, 1993, cité par P. Virilio, 1993).

9. On peut croire toucher, tenir une pomme, avoir l'impression et la sensation même de la manger, sans toutefois qu'une quelconque nourriture entre dans l'estomac.

10. Il existe des programmes combinant éducation et loisirs, les fameux edutainment programmes (education and entertainment), en CD-ROM (Compact Disk - Read Only Memory) ou en vidéo, grâce auxquels, les enfants, ou même les adultes peuvent apprendre tout en s'amusant. 
Aux États-Unis, il semblerait que les enfants passent bien davantage de temps devant leurs micro-ordinateurs qu'à l'école (R. de La Baume, J.-J. Bertolus, 1995).

11. Si, par exemple, il se met à pleuvoir dans un village «soleil », les G.M. sont complètement dépendants des G.O. pour tout ce qui concerne leur divertissement, étant donné que tout est prévu pour vivre et s'amuser à l'extérieur et qu'ils sont soudain tous obligés de vivre et s'amuser à l'intérieur où aucun équipement n'est prévu à cet effet.

12. Le terme «machine» étant pris dans son sens le plus large, celui d'un «dispositif opératoire » (A. Bressand, C. Distler, 1995).

\section{RÉSUMÉS}

Les nouvelles technologies et la réseautisation (utilisation du concept même des réseaux dans l'organisation spatiale) généralisée sont à l'origine d'un gain de temps considérable, qui pourrait être la cause de l'augmentation du temps de loisirs dans l'avenir et du développement de réseaux de loisirs. Les particularités locales combinées des sites naturels et culturels seraient alors l'attrait principal pour la consommation du temps de loisirs "réels» dans ces sites "authentiques", par opposition (ou complémentarité) avec les loisirs virtuels amenés directement aux consommateurs. Cette situation reste dans le domaine de la science-fiction et ne peut être placée que dans un futur lointain; un futur où la planète entière aura résolu les problèmes d'inégalités économiques, territoriales et culturelles; où chacun de ses habitants, indépendamment de ses origines ou statut socioprofessionnel, grâce à son travail lié, pourra bénéficier des mêmes « privilèges » pendant ses longs temps libres.

New technologies and networks are responsible for a substantial time-saving, which could be the cause of increased leisure in the future and the development of leisure networks. The combination of specific local features of natural and cultural sites would then be the principal appeal for a « real » leisure time in « authentic " places in contrast to or complementary to the virtual ones directly offered by computers. This situation belongs to the realm of science fiction and can only be conceived in a distant future, a future where the whole planet will have resolved its spatial, economic and cultural inequalities, where all inhabitants, independently of their origin and status, could enjoy the same « privileges » during their long leisure time.

Die neuen Technologien und die allgemeine Netzverdichtung sind die Ursachen eines beträchtlichen Zeitgewinns, der wiederum der Grund für die Verlängerung der Freizeit in Zukunft und für die Entwicklung von Freizeitnetzen sein könnte. Die örtlichen kombinierten Besonderheiten natürlicher und kultureller Stätten wären so der hauptsächliche Anreiz für den «reellen» Freizeitverbrauch an diesen «authentischen» Stätten - im Gegensatz oder in Ergänzung zur virtuellen Freizeit, die den Konsumenten direkt geboten wird. Dies bleibt indes im Bereich der Science-fiction und kann sich nur in ferner Zukunft entwickeln ; eine Zukunft, in der unser ganzer Planet die Probleme wirtschaftlicher, territorialer und kultureller Ungleichheit gelöst haben wird; wo jeder Einwohner, unabhängig von seiner Herkunft und seinem sozioprofessionellen Status und dank seiner Arbeit die gleichen Privilegien während seiner langen Freizeit geniessen kann. 
INDEX

Schlüsselwörter : Freizeit, Netzwerke, neue Technologien, virtueller Raum

Mots-clés : espace virtuel, loisirs, nouvelles technologies, réseaux, temps libre

Keywords : free time, leisure, networks, new technologies, virtual space

\section{AUTEUR}

\section{HÉLÈNE HANIOTOU}

Laboratoire «Image et Ville » - UPRES-A 7011 CNRS - Université Louis-Pasteur - 3, rue de l'Argonne 67000 Strasbourg 